\title{
Constructions of Finite Groups
}

\author{
Yongbin Qin \\ Department of Computer Science \\ Guizhou University (550025) \\ Guiyang, China \\ ybqin@foxmail.com, 18085008039
}

\author{
Haiyue Zhang, Daoyun Xu \\ Department of Computer Science \\ Guizhou University (550025) \\ Guiyang, China
}

\begin{abstract}
Y-group (as a matrix) in [1] presents a new representation of finite algebra systems. A complete Y-group decides a finite group, and the computation table of a finite group is a complete $\mathrm{Y}$-group. Ones can comprehend deeply constructions of finite groups based on geometric properties of matrixes. In this paper, we analyse inherent connections between the three (ordinary finite group, permutation group, and matrix group for ordinary multiplication), and investigate some methods for constructing finite groups based on geometry (or structure) properties of matrixes. It is helpful for classifying and decomposing finite groups.
\end{abstract}

Keywords-Finite group; CY-matrix; permutation; geometry method; construction; classification

\section{INTRODUCTION}

Let $G=\left\{g_{1}, \cdots, g_{n}\right\}$ be a finite group and let $g_{1}=e$ be the unit element of $G$, then for any $g_{i} \in G, g_{i} G=\left\{g_{i} g_{1}, \cdots\right.$ ,$\left.g_{i} g_{n}\right\}=G$. The operation table on $G$ can be represented as a $n \times n$ matrix $M_{G}=\left(g_{i, j}\right)$, where $g_{i, j}=g_{i} g_{j}$ for $1 \leq i, j \leq n$. The matrix $M_{G}$ has the following basic properties:

(1) Each element $a$ in $G$ occurs exactly once in each row (column) of $M_{G}$. Then, each element $a$ in $\mathrm{G}$ defines a permutation matrix on $G$.

(2) For any a $2 \times 2$ submatrix $\left(\begin{array}{ll}a & b \\ c & d\end{array}\right)$ in $M_{G}$, shortly for a block, anyone can be decided only other three. i.e., $M_{G}$ associates with a function $F: G^{3} \rightarrow G$ satisfying the constraints: $F(b, a, c)=d \Leftrightarrow F(d, b, a)=c \Leftrightarrow F(a, c, d)=b \Leftrightarrow$ $F(c, d, b)=a$.

This characterization is called as "Four Endpoint Rule" (FER) in [1].

(3) By changing properly order of rows (or columns) of $M_{G}$, we can get a new matrix $M_{G(e)}=\left(g_{i, j}^{\prime}\right)$ such that $g_{i, j}^{\prime}=$ $e$ for $i=1,2, \cdots, n$, i.e., the unit element $e$ is located at main diagonal line of $M_{G(e)}$, such matrix is called normal matrix. Thus, the operation $a * b=c$ in $G$ can be represented by a $\operatorname{block}\left(\begin{array}{ll}e & b \\ a & c\end{array}\right)$ in $M_{G(e)}$.

Clearly, for any fixed $e^{\prime} \in G$, we get a new matrix $M_{G\left(e^{\prime}\right)}$ from $M_{G}$ by the same method, and $M_{G(e)}$ decides a new group $G^{\prime}$ with the unit element $e^{\prime}$, the operation $a \Delta b=d$, denoted by $(a * b)_{e}$, in $G^{\prime}$, is decided by the block $\left(\begin{array}{ll}e^{\prime} & b \\ a & d\end{array}\right)$ in $M_{G\left(e^{\prime}\right)}$. Clearly, $G^{\prime}$ is isomorphic to $\mathrm{G}$.
It defines a new group $G^{\prime}$ with the unit element $a$, and $G^{\prime}$ is isomorphic to $\mathrm{G}$.

The matrix $M_{G}$ can be represented by a coordinate function $\eta_{G}:[n] \times[n] \rightarrow G$, where $[n]=\{1,2, \cdots, n\}$. $\eta_{G}(i, j)=a$ means assigning the coordinate $(i, j)$ to a. It is seen that if $\mathrm{G}$ is a group, then there are exactly $\mathrm{n}$ coordinates assigned to $\mathrm{a}$, and these coordinates have not same rowcoordinates or column-coordinates. The characterization function of set of these coordinates is presented as a $n \times n$ (0/1)-matrix $\chi_{a}^{M_{G}}$

For any normal matrix $M_{G(e r)}$, associating a coordinate function $\eta_{e^{\prime}}, \chi_{a}^{M_{G\left(e^{\prime}\right)}}(i, j)=1 \Leftrightarrow \eta_{e^{\prime}}(i, j)=a$. It is easy to show that $\chi_{a}^{M_{G\left(e^{\prime}\right)} *} \chi_{b}^{M_{G\left(e^{\prime}\right)}}=\chi_{d}^{M_{G\left(e^{\prime}\right)}}$ (multiplication of matrixes) if and only if there is the block $\left(\begin{array}{ll}e^{\prime} & b \\ a & d\end{array}\right)$ in $M_{G(e \prime)}$.

Note that the characterization matrix $\chi_{a}^{M_{G\left(e^{\prime}\right)}}$ is a permutation matrix $P_{\pi_{a}}$,on $[\mathrm{n}]$, where $\pi_{\alpha}(i)=j \Leftrightarrow \chi_{a}^{M_{G\left(e^{\prime}\right)}}(i, j)=1$ $\Leftrightarrow \eta_{e}(i, j)=a$. Thus, we have the following table of relations:

\begin{tabular}{|c|c|c|c|}
\hline groups & $\begin{array}{c}\text { ordinar } \\
\text { y group }\end{array}$ & $\begin{array}{c}\text { group for } \\
\text { permutatio } \\
\boldsymbol{n} \text { s }\end{array}$ & group f or matrixes \\
\hline elements & $\alpha$ & $\pi_{a}$ & $\chi_{a}^{M_{G(e)}}$ \\
\hline operations & $\alpha b$ & $\pi_{a} \circ \pi_{b}$ & $\chi_{a}^{M_{G(e)} * \chi_{b}^{M_{G(e)}}}$ \\
\hline results & $\alpha b=c$ & $\begin{array}{l}\pi_{a} \circ \pi_{b} \\
=\pi_{c}\end{array}$ & $\chi_{a}^{M_{G(e)} * \chi_{b}^{M_{G(e)}}=\chi_{c}^{M_{G(e)}}}$ \\
\hline
\end{tabular}

We think for a finite group $G=(\Omega, *)$, the matrix $M_{G}$ is a geometric representation of basic set $\Omega$ in G, and FER in $M_{G(e)}$ describe some constraints of geometry structures on $\Omega$, and the constraints come from the operation "*”.

A reverse idea is whether or not we can define originally finite algebra systems from geometry structures satisfying constraints (FER) on $\Omega$. This idea comes from the author of reference [1].

In [1], the author introduces two basic concepts, Y-group and CY-group (complete Y-group). Simply, a Y-group on $\Omega$ is a $p \times q$ matrix $\mathrm{M}$, shortly for $\mathrm{Y}$-matrix, if $\mathrm{M}$ satisfies FER. $\mathrm{M}$ is presented as a function $\eta:[p] \times[q] \rightarrow \Omega$. In a Y-matrix $\mathrm{M}$, each element $\alpha$ in $\Omega$ occurs at most once in each row 
(column) of $\mathrm{M}$. The size of $\mathrm{M}$ is defined by $k / \min \{p, q\}$, where $\mathrm{k}$ is the number of elements occurring in $M$ of $\Omega$. If $\mathrm{k}=1$, then $\mathrm{M}$ is called as complete, shortly for CY-matrix. If a Y-matrix $\mathrm{M}$ is complete, then $p=q=k$, and each element $a$ in $\Omega$ occurs exactly once in each row (column) of $\mathrm{M}$. The important property is that Y-matrix satisfies FER.

Thus, in this paper, we discuss mainly matrixes with the property FER in languages of matrixes. We will find that between CY-matrixes and finite groups are one-to-one. Between the three, ordinary finite group, permutation group, and matrix group for ordinary multiplication, have natural inherent connections.

The geometry properties of matrixes will be useful for constructing finite groups, classifying and decomposing of finite groups. In this paper, a lot of ideas are original or citing from [1], and constructing, classifying and decomposing for finite groups are classical and ancient problems, we cite a few of references except necessary. The relevant references can be seen in $[2,3,4,5,6,7,8]$.

In paper, we present some methods for constructing finite groups based on geometry (or structure) properties of matrixes. It is helpful for classifying and decomposing finite groups.

\section{FINITE EUCLIDEAN SPACES}

For given sets $N_{n_{i}}=1,2, \cdots, n_{i}(i=1,2, \cdots k)$, the Descartes product $N_{n_{1}} \times \cdots \times N_{n_{k}}$ is called the $k$-dimension finite Euclidean space (see [9]). Specially, 2-dimension finite Euclidean space is called as finite Euclidean plane. The finite Euclidean plane $N_{p} \times N_{q}$ can be represented by a $p \times q$ matrix $S=((i, j))_{p \times q}$, where the original point is defined to $(1,1)$, and the eatery $(i, j)$ is a coordinate point.

For a finite set $\Omega=\left\{a_{1}, \cdots a_{n}\right\}$ (in short, $\Omega=\{1, \cdots, n\}$ ), the partial function $\eta: \subseteq N_{p} \times N_{q} \rightarrow \Omega$ is taken as assigning coordinates to elements in $\Omega$, called a representation of $\Omega$. Normally, we define the function $\eta$ as a total function, and take $p=q$.

A function $\eta: \subseteq N_{p} \times N_{q} \rightarrow \Omega$ can be represented as a matrix $=(\eta(i, j))_{p \times q}$, denote as $\llbracket \eta \rrbracket$.

In the coordinate matrix $S=((i, j))_{p \times q}$, any a $2 \times 2$ submatrix (called a block) consists of four points $\left\{(i, j),\left(i, j^{\prime}\right),\left(i^{\prime}, j\right),\left(i^{\prime}, j^{\prime}\right)\right\}$, i.e. a block depends on at most four parameters $i, j, i^{\prime}, j^{\prime}$, or at most three points $\left\{(i, j),\left(i, j^{\prime}\right),\left(i^{\prime}, j\right)\right\}$. By the function $\eta$, a block in $S$ is projected to a block in $\llbracket \eta \rrbracket$.

$$
\left(\begin{array}{cc}
(i, j) & \left(i, j^{\prime}\right) \\
\left(i^{\prime}, j\right) & \left(i^{\prime}, j^{\prime}\right)
\end{array}\right) \underset{\eta}{\rightarrow}\left(\begin{array}{ll}
\eta(i, j) & \eta\left(i, j^{\prime}\right) \\
\eta\left(i^{\prime}, j\right) & \eta\left(i^{\prime}, j^{\prime}\right)
\end{array}\right)
$$

The point $\left(i^{\prime}, j^{\prime}\right)$ can be viewed as the result by replacing the coordinates from the point $(i, j)$ step by step.

In the above matrix $\eta \rrbracket$, we can observe an interesting result: any two blocks $\left(\begin{array}{cc}\eta(i, j) & \eta\left(i, j^{\prime}\right) \\ \eta\left(i^{\prime}, j\right) & \eta\left(i^{\prime}, j^{\prime}\right)\end{array}\right)$ and $\left(\begin{array}{cc}\eta(s, t) & \eta\left(s, t^{\prime}\right) \\ \eta\left(s^{\prime}, t\right) & \eta\left(s^{\prime}, t^{\prime}\right)\end{array}\right)$, if any values of three points are same at corresponding positions, then the value of fourth point is same.
The constraint relation is called shortly as "Four Endpoints Rule (FER)".

If the function $\eta: N_{p} \times N_{q} \rightarrow \Omega$ satisfies FER, we can introduce a function $\mathrm{F}: \Omega^{3} \rightarrow \Omega$ defined by $F(b, a, c)=d$, where the $2 \times 2$ matrix $\left(\begin{array}{ll}a & c \\ b & d\end{array}\right)$ is a block in the matrix $\llbracket \eta \rrbracket$. The general definition is from [1].

Definition 1 A 5-tuple $\left[\Omega, N_{p}, \eta, F, k\right]$ is called k-dimension Y-group, where

(1) $\Omega=\{1,2, \cdots, n\}$ is a finite set, $N_{p}=\{1,2, \cdots, p\}$ is a finite set of index.

(2) $\eta: N_{p}^{k} \rightarrow \Omega$ is a representation function, $\left(i_{1}, \cdots\right.$ ,$\left.i_{k}\right) \mapsto \eta\left(i_{1}, \cdots, i_{k}\right)$.

(3) The function $F: \Omega^{k+1} \rightarrow \Omega$ satisfies the condition on replacing coordinates: for any $i_{1}, \cdots, i_{k}, j_{1}, \cdots, j_{k} \in$ $N_{p}, F\left(\eta\left(i_{1}, \cdots, i_{k}\right), \eta\left(j_{1}, i_{2} \cdots, i_{k}\right), \cdots, \eta\left(i_{1}, j_{2}, \cdots, i_{k}\right), \cdots\right.$ ,$\eta\left(i_{1}, i_{2}, \cdots, i_{k-1}, j_{k}\right)=\eta\left(j_{1}, j_{2}, \cdots, j_{k}\right)$.

If the function $\eta$ is a full projection and for any $1 \leq s \leq k$ and any $t, t^{\prime} \in N_{p}, t \neq t^{\prime}$ implies $\eta\left(i_{1}, \cdots, i_{s-1}, t, i_{s+1}, \cdots\right.$ ,$\left.i_{k}\right) \neq \eta\left(i_{1}, \cdots, i_{s-1}, t^{\prime}, i_{s+1}, \cdots, i_{k}\right)$, then call the system $\left[\Omega, N_{p}, \eta, F, k\right]$ complete Y-group, in short CY-group.

In this paper, we focus on the case $\mathrm{k}=2$, the complete $\mathrm{Y}$ group $\left[\Omega, N_{p}, \eta, F, 2\right]$ is simplified into 4-tuple $\left[\Omega, N_{p}, \eta, F\right]$, and the condition (3) in the definition is written as $F\left(\eta\left(i^{\prime}, j\right), \eta(i, j), \eta\left(i, j^{\prime}\right)=\eta\left(i^{\prime}, j^{\prime}\right)\right.$ for any $i, j, i^{\prime}, j^{\prime} \in N_{p}$. It corresponds to a block in the matrix $\llbracket \eta \rrbracket$ :

$$
\begin{array}{r}
\left(\begin{array}{cc}
\eta(i, j) & \eta\left(i, j^{\prime}\right) \\
\eta\left(i^{\prime}, j\right) & \eta\left(i^{\prime}, j^{\prime}\right)
\end{array}\right) \text {, or }\left(\begin{array}{cc}
\eta\left(i^{\prime}, j\right) & \eta\left(i^{\prime}, j^{\prime}\right) \\
\eta(i, j) & \eta\left(i, j^{\prime}\right)
\end{array}\right) \\
\text { or }\left(\begin{array}{cc}
\eta\left(i, j^{\prime}\right) & \eta(i, j) \\
\eta\left(i^{\prime}, j^{\prime}\right) & \eta\left(i^{\prime}, j\right)
\end{array}\right), \text { or }\left(\begin{array}{ll}
\eta\left(i^{\prime}, j^{\prime}\right) & \eta\left(i^{\prime}, j\right) \\
\eta\left(i, j^{\prime}\right) & \eta(i, j)
\end{array}\right)
\end{array}
$$

In the finite Euclidean space, $\mathrm{S}=((i, j))_{p \times q}$, we introduce similarly the notation on lines. The set of points $(1, b)$, $(2, a b(\bmod q)), \cdots,(p,(p-1) a b(\bmod q))$ is called as a line in $\mathrm{S}$, if $b, a b(\bmod q), \cdots,(p-1) a b(\bmod q)$ are distinct.

\section{Basic properties of complete Y-group $\left[\Omega, \boldsymbol{N}_{\boldsymbol{p}}, \boldsymbol{\eta}, \boldsymbol{F}\right]$}

In the section, we consider some basic and important properties of the complete Y-group $\left[\Omega_{p}, N_{p}, \eta, F\right]$, where $|\Omega|=p$.

By the completeness and FER, it is easy to prove the following properties.

Property 1 (1) The function $\mathrm{F}$ holds the composite rule, i.e., for any $a, b, c, d, e, f \in \Omega$

(1.1) $F(F(a, c, f), f, d)=F(a, c, d)$, corresponding the submatrix in $\llbracket \eta \rrbracket$

$$
\left(\begin{array}{lll}
a & e & b \\
c & f & d
\end{array}\right)
$$

(1.2) $F(a, e, F(e, c, d))=F(a, c, d)$, corresponding the submatrix in $\llbracket \eta \rrbracket$ 


$$
\left(\begin{array}{ll}
a & b \\
e & f \\
c & d
\end{array}\right)
$$

(2) For any $a, b \in \Omega, F(a, a, b)=b, F(a, b, b)=a$.

Property 2 In a complete Y-group $\left[\Omega_{p}, N_{p}, \eta, F\right]$, each element $a$ in $\Omega$ occurs exactly once in each row (column) in matrix $\llbracket \eta \rrbracket$.

Property 3 For a given complete Y-group $\left[\Omega_{p}, N_{p}, \eta, F\right]$, the system $\left[\Omega_{p}, N_{p}, \eta^{\prime}, F^{\prime}\right]$ is a new system defined by arranging the order of rows (or columns) of $\llbracket \eta \rrbracket$, then $F=F^{\prime}$, i.e., the function $F$ is an invariant under arranging the order of rows (or columns).

In fact, the diagonal relationship between elements in $\llbracket \eta \rrbracket$ is fixed under rearranging the order of rows (or columns) of $\llbracket \eta \rrbracket$.

Based on Property 3, we can introduce the formal form of the matrix $\llbracket \eta \rrbracket$. For any fixed element $a$ in $\Omega$, we can adjust $a$ to the main diagonal of a matrix.

The normal form is similar to the table of computation for a finite group, where the element $a$ on the main diagonal of the matrix is equivalent to the unit element in group.

Let $[n]$ denote the set $\{1,2, \cdots, n\}$, a permutation $\pi=$ $\left(\begin{array}{cccc}1 & 2 & \cdots & n \\ \pi(1) & \pi(2) & \cdots & \pi(n)\end{array}\right)$ on $[n]$ can be shown a $(0 / 1)-$ matrix $\mathrm{Col}_{\pi}$, where, $\mathrm{Col}_{\pi}(i, j)=1$ if $i=\pi(j)$, otherwise $\operatorname{Col}_{\pi}(i, j)=0$. Clearly, $\operatorname{Col}_{\pi^{-1}}=\operatorname{Col}_{\pi}^{-1}=\left(\operatorname{Col}_{\pi}\right)^{T}$ for any permutation $\pi$, denote $\operatorname{Row}_{\pi}=\operatorname{Col}_{\pi}^{-1}$, i.e., $\operatorname{Row}_{\pi}=$ $\mathrm{Col}_{\pi^{-1}}$, where $\pi^{-1}$ is the inverse transformation of $\pi$, and the matrix $A^{T}$ is the transpose of matrix $A$.

For a matrix $A=\left(a_{i, j}\right)$, we write $A$ as $[A(1,:), \cdots, A(n$, : )$]^{T}$ in arrays of rows, or $[A(:, 1), \cdots, A(:, n)]$ in arrays of columns. Then, we have the following relations:

$$
A \cdot \operatorname{Col}_{\pi}=[A(:, 1), \cdots, A(:, n)] \cdot \operatorname{Col}_{\pi}=
$$
$[A(:, \pi(1)), \cdots, A(:, \pi(n)]$.

$$
\operatorname{Col}_{\pi}^{-1} \cdot A=\operatorname{Row}_{\pi} \cdot[A(1,:), \cdots, A(n,:)]^{T}=
$$
$[A(\pi(1),:), \cdots, A(\pi(n),:)]^{T}$.

Therefore, we have that

Property 4 For a given complete Y-group $\left[\Omega, N_{p}, \eta, F\right]$, the representation matrix is $\llbracket \eta \rrbracket$, then for any fixed element $a \in$ $\Omega$, there is a permutation $\pi$ on $[p]$, such that the system $\left[\Omega_{p}, N_{p}, \eta_{a}, F\right]$ is a complete Y-group, where the element $a$ is located at the main diagonal line of $\llbracket \eta_{a} \rrbracket$, and $\llbracket \eta_{a} \rrbracket=R o w_{\pi} \cdot \llbracket \eta \rrbracket$.

Please note that the function $F$ is an invariant up to permutations. In [1], the author presents a directly definition of complete Y-group, if the function $F: \Omega^{3} \rightarrow \Omega$ on $\Omega$ holds the following properties, then $F$ decides a complete $\mathrm{Y}$-group.

(1) FER condition: for any a, b, c, d $\in \Omega$, presenting as $\operatorname{block}\left(\begin{array}{ll}a & c \\ b & d\end{array}\right)$

$F(b, a, c)=d \Leftrightarrow F(a, c, d)=b \Leftrightarrow F(c, d, b)=a \Leftrightarrow$ $F(d, c, a)=b$.

(2) Composite condition: for any $a, b, c, d, e, f \in \Omega$
$F(F(a, c, f), f, d)=$

$F(a, c, d)$, and $F(a, e, F(e, c, d))=F(a, c, d)$.

In fact, FER implies the composite condition.

\section{COMPLETE Y-GROUPS AND FINITE GROUPS}

In [1], the author presents the following results:

(1) A finite group defines a complete Y-group $\left[\Omega, N_{p}, \eta, F\right]$.

complete Y-group $\left[\Omega, N_{p}, \eta, F\right]$ can decide a usual finite group. For any fixed element $\theta \in \Omega$, define an operation $*, a * b=$ $F(a, \theta, b)$, it corresponds to a block $\left(\begin{array}{cc}\theta & b \\ a & F(a, \theta, b)\end{array}\right)$.

We now introduce another method defining a finite group from a complete Y-group. Basic idea comes from [1].

For a given complete Y-group $\left[\Omega, N_{p}, \eta, F\right]$, we have a representation square matrix $\llbracket \eta \rrbracket$ of p-order. For an element $a \in$ $\Omega$, define a $0 / 1$ square matrix $\chi_{\alpha}^{\llbracket \eta \rrbracket}$ of p-order as follows: $\chi_{\alpha}^{\llbracket \eta \rrbracket}(i, j)=\left\{\begin{array}{cc}1 & \eta(i, j)=a \\ 0 & \text { o.w. }\end{array}\right.$.

The matrix $\chi_{\alpha}^{\llbracket \eta \rrbracket}$ is called the characterization matrix of $a$.

Lemma 1 For a given complete Y-group $\left[\Omega, N_{p}, \eta, F\right]$ and a fixed element $\theta$ in $\Omega, \llbracket \eta \rrbracket_{\theta}$ is a formal matrix on $\theta$ from $\llbracket \eta \rrbracket$, i.e., the element $\theta$ is located at main diagonal of the matrix by rearranging order of rows in $\llbracket \eta \rrbracket$, then for any block $\left(\begin{array}{ll}\theta & b \\ a & c\end{array}\right)$ in $\llbracket \eta \rrbracket_{\theta}$, we have that $\chi_{a}^{\llbracket \eta \rrbracket_{\theta}} * \chi_{b}^{\llbracket \eta \rrbracket_{\theta}}=\chi_{c}^{\llbracket \eta \rrbracket_{\theta}}$, where the operation "*” is the usual multiplication of matrix.

Proof: Let $\left[\Omega, N_{p}, \eta, F\right]$ be a complete Y-group, then $|\Omega|=$ $p$ and the function $\eta: \Omega \rightarrow N_{p}$ can be represented as a $\mathrm{p} \times \mathrm{p}$ matrix $\llbracket \eta \rrbracket$, where the matrix $\llbracket \eta \rrbracket$ satisfies FER. For a given element $\theta$ in $\Omega$, the formal matrix is denoted as $\llbracket \eta \rrbracket_{\theta}$,corresponding to a function $\eta_{\theta}: \Omega \rightarrow N_{p}$, and $\llbracket \eta \rrbracket_{\theta}$ satisfies FER. $\left(\llbracket \eta \rrbracket_{\theta}=\llbracket \eta_{\theta} \rrbracket\right)$.

Let $\Omega=\left\{\theta, a_{2}, \cdots, a_{p}\right\}\left(a_{1}=\theta\right)$, and $\eta_{\theta}(1,1)=\theta$, $\eta_{\theta}(1,2)=a_{2}, \cdots, \eta_{\theta}(1, p)=a_{p}$. Based on Property 2 in Section 3, for any $a \in \Omega$, the matrix $\chi_{a}^{\llbracket \eta \rrbracket_{\theta}}$ is a permutation matrix, corresponding to a permutation $\pi_{a}$, i.e., $\chi_{a}^{\llbracket \eta \rrbracket_{\theta}}=\operatorname{Col}_{\pi_{a}}$.

For any $a \in \Omega$ and each $1 \leq k \leq p$, we have two coordinate arguments, a row argument $r_{a}^{[k]}$ and a column argument $c_{a}^{[k]}$, corresponding to $\chi_{a}^{\llbracket \eta \rrbracket_{\theta}}\left(k, c_{a}^{[k]}\right)=1$ and $\chi_{a}^{\llbracket \eta \rrbracket_{\theta}}\left(r_{a}^{[k]}, k\right)=1$.

For any block $\left(\begin{array}{ll}\theta & b \\ a & c\end{array}\right)$ in $\llbracket \eta \rrbracket_{\theta}$ and each $1 \leq k \leq p$, we have the following relations: $\eta_{\theta}(k, k)=\theta, \eta_{\theta}\left(k, c_{b}^{[k]}\right)=$ $b, \eta_{\theta}\left(r_{a}^{[k]}, k\right)=a, \eta_{\theta}\left(r_{a}^{[k]}, c_{b}^{[k]}\right)=c$.

For any $a \in \Omega$, the characterization matrix $\chi_{a}^{\llbracket \eta \rrbracket_{\theta}}$ has the property that for each $1 \leq k \leq p, \chi_{a}^{\llbracket \eta \rrbracket_{\theta}}(i, k)=1 \Leftrightarrow i=$ $r_{a}^{[k]}$ and $\chi_{b}^{\llbracket \eta \rrbracket_{\theta}}(k, j)=1 \Leftrightarrow j=c_{b}^{[k]}$. 
Let $\quad \chi_{a}^{\llbracket \eta \rrbracket_{\theta}}=\left(a_{i, j}\right)_{p \times p}, \chi_{b}^{\llbracket \eta \rrbracket_{\theta}}=\left(b_{i, j}\right)_{p \times p} \quad$ and $\quad \chi_{c}^{\llbracket \eta \rrbracket_{\theta}}=$ $\left(c_{i, j}\right)_{p \times p}$, and denote $\left(a_{i, j}\right)_{p \times p} *\left(b_{i, j}\right)_{p \times p}=\left(d_{i, j}\right)_{p \times p}$, then $d_{i, j}=\sum_{k=1}^{p} a_{i, k} b_{k, j}$ in the usual multiplication of matrix for each pair $1 \leq i, j \leq p$.

We have that $0 \leq d_{i, j} \leq 1$, otherwise, there exist $k, k^{\prime}(1 \leq$ $\left.k \neq k^{\prime} \leq p\right)$ such that $a_{i, k} b_{k, j}=a_{i, k^{\prime}} b_{k^{\prime}, j}=1$. It implies that $a_{i, k}=a_{i, k^{\prime}}=1$. It contradicts with Property 2 .

Further, we have that $d_{i, j}=1$ if and only if there is the unique $k_{*}$ such that $\left(a_{i, k_{*}}=1\right) \wedge\left(b_{k_{*}, j}=1\right)$ Please note that $\left(a_{i, k_{*}}=1\right) \wedge\left(b_{k_{*}}=1\right) \Leftrightarrow\left[\left(i=r_{a}^{\left[k_{*}\right]}\right) \wedge\left(j=c_{b}^{\left[k_{*}\right]}\right)\right] \quad, \quad$ it corresponds to a block in $\llbracket \eta \rrbracket_{\theta}$.

$$
\left(\begin{array}{cc}
\left(k_{*}, k_{*}\right) & \left(k_{*}, c_{b}^{\left[k_{*}\right]}\right) \\
\left(r_{a}^{\left[k_{*}\right]}, k_{*}\right) & \left(r_{a}^{\left[k_{*}\right]}, c_{b}^{\left[k_{*}\right]}\right)
\end{array}\right) \rightarrow \eta_{\theta}\left(\begin{array}{cc}
\theta & b \\
a & c
\end{array}\right) .
$$

It means that $\left(d_{i, j}=1\right) \Leftrightarrow\left(d_{i, j}=\sum_{k=1}^{p} a_{i, k} b_{k, j}=\right.$

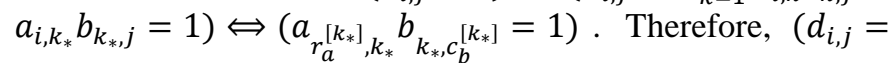
1) $\Leftrightarrow\left(c_{i, j}=1\right)$, i.e., $d_{i, j}=c_{i, j}$.

Therefore, we have that

Theorem 1 For a given complete system $\left[\Omega, N_{p}, \eta, F\right]$ and a fixed element $\theta$ in $\Omega$, let $M=\left\{\chi_{a}^{\llbracket r \rrbracket_{\theta}}: a \in \Omega\right\}$ be a set of (0/1)matrixes of p-order, then $(M, *)$ is a group, where the binary operation "*" is the usual multiplication of matrix, and $\chi_{\theta}^{\llbracket \eta \rrbracket_{\theta}}$ is the unit element in the group.

Generally, let $\left[\Omega, N_{p}, \eta, F\right]$ be a complete Y-group, we can decide the function $F: \Omega^{3} \rightarrow \Omega$ from the matrix representation $\llbracket \eta \rrbracket$ of $\eta$, and $F$ is an invariant, i.e., it is independent on the order of rows (columns) in $\llbracket \eta \rrbracket$. For any fixed element $\theta$ in $\Omega$, we can get a normal function $\eta_{\theta}: N \times N_{p} \rightarrow \Omega$ and a normal matrix $\llbracket \eta \rrbracket_{\theta}=\llbracket \eta_{\theta} \rrbracket$.

Based on $\llbracket \eta_{\theta} \rrbracket$, we rewrite $F(a, \theta, b)$ as $F_{\theta}(a, b)$.The function $F_{\theta}: \Omega^{2} \rightarrow \Omega$ defines a binary operation on $\Omega$ by a block $\left(\begin{array}{ll}\theta & b \\ a & c\end{array}\right)$ in $\llbracket \eta_{\theta} \rrbracket:\left(a *_{\theta} b\right)=(a * b)_{\theta}=F_{\theta}(a, b)=c$.

If there are two blocks $\left(\begin{array}{ll}\theta & b \\ a & c\end{array}\right)$ and $\left(\begin{array}{ll}\theta & a \\ b & c^{\prime}\end{array}\right)$ in $\llbracket \eta_{\theta} \rrbracket$ such that $c \neq c^{\prime}$, then call that the operation is not exchangeable. Otherwise, the operation is exchangeable.

There are unexchangeable complete Y-groups.

Let $\chi_{a}^{\llbracket \eta \rrbracket}$ (resp. $\chi_{a}^{\llbracket \eta_{\theta} \rrbracket}$ ) be the characterization matrix of the element $a$ from $\llbracket \eta \rrbracket$ (resp. $\left.\llbracket \eta_{\theta} \rrbracket\right)$ for any $a \in \Omega$. We get a permutation $\pi_{\alpha}$ on $N_{p}$ from $\chi_{a}^{\llbracket \eta \rrbracket}$, where $\operatorname{Col}_{\pi_{a}}=\chi_{a}^{\llbracket \eta \rrbracket}$.

We have the following relations:

(1) $\llbracket \eta_{\theta} \rrbracket=\operatorname{Row}_{\pi_{\theta}} * \llbracket \eta \rrbracket$.

(2) $\chi_{a}^{\llbracket \eta \eta_{\theta} \rrbracket}=\operatorname{Row}_{\pi_{\theta}} * \chi_{a}^{\llbracket \eta \rrbracket}$ for any $a \in \Omega$.

(3) $\chi_{a}^{\llbracket \eta_{\theta} \rrbracket} * \chi_{b}^{\llbracket \eta_{\theta} \rrbracket}=\chi_{c}^{\llbracket \eta_{\theta} \rrbracket} \Leftrightarrow \chi_{a}^{\llbracket \eta \rrbracket} * R o w_{\pi_{\theta}} * \chi_{b}^{\llbracket \eta \rrbracket}=$ $\chi_{c}^{\llbracket \eta \rrbracket} \Leftrightarrow \pi_{a} \circ \pi_{\theta}^{-1} \circ \pi_{b}=\pi_{c}$.
(4) $\llbracket \eta \rrbracket=\sum_{a \in \Omega} a \cdot \chi_{a}^{\llbracket \eta \rrbracket}$.

\section{CONSTRUCTING COMPLETE Y-GROUPS FROM PERMUTATIONS}

Let [n] denote the set $\{1,2, \cdots, n\}$, and let $\pi=$ $\left(\begin{array}{ccccc}1 & 2 & \cdots & \cdots & n \\ \pi(1) & \pi(2) & \cdots & \cdots & \pi(n)\end{array}\right)$ be a permutation on $[n]$. The permutation $\pi$ can be decomposed a set of cyclic permutation, $\pi_{1}, \cdots, \pi_{k}$, where $\pi_{i}$ is a cyclic permutation on some subsets $S_{\pi_{i}}$ of $[n]$, such that $[n]=\bigcup_{i=1}^{k} S_{\pi_{i}}$ and $\mathrm{S}_{\pi_{\mathrm{i}}} \cap \mathrm{S}_{\pi_{\mathrm{j}}}=\emptyset$ for any $\mathrm{i}$, $\mathrm{j}(1 \leq i \neq j \leq k)$. The size of $S_{\pi_{i}},\left|S_{\pi_{i}}\right|$, is called the length of cyclic permutation $\pi_{i}$. In this paper, we assume that $\left|S_{\pi_{i}}\right| \geq 2$ for each i, since the element $a$ can be deleted from $[n]$ if $\left|S_{\pi_{i}}\right|=$ $\{a\}$ for some cyclic $\pi_{i}$. Such $\pi$ is called nontrivial permutation. If $k=1$, then $\pi$ is a cyclic permutation of length $\mathrm{n}$, and it can be written as $\left(j_{1} j_{2} \cdots j_{n}\right)$, which defines an order $\left(j_{1} \prec\right.$ $j_{2} \prec \cdots \prec j_{n}$ ) on [n], where $j_{1}=\pi(1)$.

Let $\pi$ be a cyclic permutation of length $n$ on [n]. Define $\pi^{0}=I d_{[n]}$ (identical transformation), $\pi^{k+1}=\pi \circ \pi^{k}(k=$

$0,1,2, \cdots, n-1)$ and $\pi^{k}([n])=\left(\pi^{1}([n]), \cdots \cdots\right.$ , $\left.\pi^{k}([n])\right)$, then the matrix $A^{\pi}=\left(\pi^{0}([n]), \pi^{1}([n]), \cdots\right.$, $\left.\pi^{n-1}([n])\right)^{T}$ decides a complete Y-group.

We now introduce another method to define a matrix $B^{\pi}=$ $\left(b_{i, j}\right)$ based directly on FER, such that $b_{1, k}=b_{k, 1}=k(k=$ $1,2, \cdots, n)$.

Let $\pi=\left(j_{1} j_{2} \cdots \cdots j_{n}\right)$ be a cyclic permutation of length $\mathrm{n}$ on $[n]$.

(1) Set $\left(b_{1,1}, b_{1,2}, \cdots, b_{1, n}\right)=(1,2, \cdots, n)$ first row of $B^{\pi},\left(b_{j_{1}, 1}, b_{j_{1}, 2}, \cdots, b_{j_{1}, n}\right)=(\pi(1), \pi(2), \cdots, \pi(n)) \mathrm{j} 1$-th row of $B^{\pi}$ and $\left(\mathrm{b}_{1, \mathrm{j}_{1}}, \mathrm{~b}_{2, \mathrm{j}_{1}}, \cdots, \mathrm{b}_{\mathrm{n}, \mathrm{j}_{1}}\right)=(\pi(1), \pi(2), \cdots, \pi(\mathrm{n}))$ j1-th column of $B^{\pi}$.

(2) For $k=1,2, \cdots, n-1$ suppose that $\mathrm{jk}$-th column $B^{\pi}\left(:, j_{k}\right)$ of $B^{\pi}$ has been computed ,computing $\mathrm{jk}+1$-th column $B^{\pi}\left(:, j_{k+1}\right)$ of $B^{\pi}$ based on $B^{\pi}\left(:, j_{k}\right)$ by FER:

For $\in[n]-\left\{1, j_{1}\right\}$, let $a=b_{i, j_{k}}$,finding column index $c_{a}$ of a in $B^{\pi}(1,:)$.

\section{BASIC COMPLETE Y-GROUPS}

Let $\mathrm{p}$ be a natural number, e.g. $\mathrm{p}$ is a prime number, and denote $C_{p}$ as a complete Y-group defined by a cyclic permutation on $[p]=\{1,2, \cdots, p\} . C_{p}$ is described in matrix of p-order. Such matrixes of form $C_{p}$ are basic (or atomic) components constructing more complex complete Y-groups. In this section, we consider the classifying and operations about $C_{p}$.

Let $(a)=\left(a_{1}, a_{2}, \cdots, a_{p}\right)$ be a cyclic sequence of symbols associating a function next next $_{a}\left(a_{i}\right)=a_{(i+1)(\bmod p)}$, and a set $\operatorname{Sym}(a)=\left\{a_{1}, \cdots, a_{p}\right\}$, where $\mathrm{p}$ is the length of $(a)$. For $1 \leq r \leq p-1$, we call that between $\mathrm{r}$ and $\mathrm{p}$ are coprime, denoted by $(r, p)=1$, if for any $i, j(1 \leq i \neq j \leq p)$, $(i$. $r)(\bmod p) \neq(j \cdot r)(\bmod p) \quad, \quad$ or $\{1,[r(\bmod p)]+$ $1,[(2 r)(\bmod p)]+1, \cdots,[(p-1) r(\bmod p)]+1\}=$ $\{1,2, \cdots, p\}$. Such $r$ is called as Euler number of $p$. Define a set $\operatorname{Euler}(p)=\{r: r$ is a Euler number of $p\}$. Clearly, 
Euler(p) contains 1 for any $p \geq 2$, and if $\mathrm{p}$ is a prime number, then $\operatorname{Euler}(p)=\{1,2, \cdots, p-1\}$.

If $(r, p)=1$, we define recursively a cyclic matrix as follows:

(1) $C_{p, r}(1, j)=j$ for $j=1,2, \cdots, p$. 1.

(2) $C_{p, r}(i+1,1)=(\operatorname{ir})(\bmod p)+1$ for $i=1,2, \cdots, p-$

(3) $C_{p, r}(i, j+1)=\left[C_{p, r}(i, j)(\bmod p)\right]+1$ for $j=$ $1,2, \cdots, p-1$.

Clearly, if $\quad(r, p)=1 \quad$ then $\left\{C_{p, r}(1,1), C_{p, r}(2,1), \cdots, C_{p, r}(p, 1)\right\}=\{1,2, \cdots, p\}=[p] \quad$, therefore, $C_{p, r}$ is a complete Y-matrix.

The matrix $C_{p, r}=\left(c_{i, j}\right)_{p \times p}$ will be viewed as a basic model (or matrix of index) of complete Y-group. For a set $\Omega=$ $\left\{a_{1}, a_{2}, \cdots, a_{p}\right\}$ and a cyclic sequence $(a)=<$ $a_{1}, a_{2}, \cdots, a_{p}>$ of symbols. $C_{p, r}(a)$ defines a complete $\mathrm{Y}$ group on $\Omega$ associating with a function $\eta: N_{p} \times N_{p} \rightarrow \Omega$, where $\eta(1, j)=a_{j}, \eta(i, 1)=a_{[r(i-1)](\bmod p)+1}$ and $\eta(i, j)=$ $\operatorname{next}_{a}(\eta(i, j-1))$ for $1 \leq i \leq p, 2 \leq j \leq p$.

Lemma 2 Assume that $p \geq 2$ and $(r, p)=1$, then $\mathrm{C}_{\mathrm{p}, \mathrm{r}}=$ Row $_{\pi_{\mathrm{p}, \mathrm{r}}} * \mathrm{C}_{\mathrm{p}, 1}$, where $\pi_{p, r}(1)=r+1, \pi_{p, r}(j+1)=$ $\left(\pi_{p, r}(j)+r\right)(\bmod p)+1$ for $j=1,2, \cdots, p-1$, the number $r$ is called as rotation parameter of rows in $C_{p, 1}$.

Lemma 3 [1] Let $(a),(b),(c)$ and $(d)$ be four cyclic sequences of symbols of length $p$, where $\operatorname{Sym}(a) \cap \operatorname{Sym}(b)=$ $\emptyset, \operatorname{Sym}(a) \cap \operatorname{Sym}(c)=\emptyset$, and $\operatorname{Sym}(b) \cap \operatorname{Sym}(d)=\emptyset$. Then, the matrix $M=\left(\begin{array}{ll}C_{p, r}(a) & C_{p, s}(b) \\ C_{p, u}(c) & C_{p, v}(d)\end{array}\right)$ is a Y-matrix, if and only if $r v \equiv s u(\bmod p)$, where $(r, p)=(s, p)=(u, p)=$ $(v, p)=1$.

If $\operatorname{Sym}(a)=\operatorname{Sym}(d)$ and $\operatorname{Sym}(b)=\operatorname{Sym}(c)$, then $M$ is a CY-matrix when $M$ is a Y-matrix.

For natural numbers $p \geq 2, q \geq 2$, let $\left(a_{1}\right), \cdots,\left(a_{q}\right)$ be $\mathrm{q}$ distinct cyclic sequences of symbols of length $p$, i.e., $\operatorname{Sym}\left(a_{i}\right) \cap \operatorname{Sym}\left(a_{j}\right)=\emptyset$ for $i \neq j$. Define a $q \times q$ matrix $R=\left(r_{i, j}\right)$ of rotation parameters of rows in $C_{p, 1}$, where $\left(r_{i, j}, p\right)=1$ for any $1 \leq i, j \leq q$, such that for any $2 \times 2$ block $\left(\begin{array}{ll}r & s \\ u & v\end{array}\right)$ in $R, r v \equiv \operatorname{su}(\bmod p)$.

We view $\left(a_{i}\right)$ as a symbol, and fix a cyclic sequence $(\vec{a})=$ $\left(\left(a_{1}\right), \cdots,\left(a_{q}\right)\right)$, take a cyclic matrix $C_{q, r^{*}}\left(\left(r^{*}, q\right)=1\right)$ as a model getting matrix $C_{q, r^{*}}(\vec{a})$. Combining $R$ with $C_{q, r^{*}}(\vec{a})$, we can construct a complete Y-matrix $M=\left(M_{i, j}\right)$, where $M_{i, j}$ is the form of $C_{p, r_{i, j}}\left(a_{t}\right), C_{q, r^{*}}(i, j)=\left(a_{t}\right)$ and $R(i, j)=r_{i, j}$.

\section{CONSTRUCTION OF FINITE GROUPS}

For $p, q \geq 2$ and the cyclic sequence $(p)=(1,2, \cdots, p)$, we define a cyclic sequence $(p)_{b}=((b-1) p+1,(b-1) p+$ $2, \cdots, b p)$ for $1 \leq b \leq q$. Let $\mathrm{R}$ be a $q \times q$ matrix on the set $\operatorname{Euler}(p)$ of Euler number with respect to $p$, such that for any
$2 \times 2$ block $\left(\begin{array}{ll}r & s \\ u & v\end{array}\right)$ in $R, r v \equiv \operatorname{su}(\bmod p)$, it is called the diagonal congruence w.r.t. $p$.

We now define two products of cyclic matrixes, $C_{p, r} \otimes$ $C_{q, r}$ and $C_{p, 1} \otimes_{R} C_{q, r}$, where $r^{\prime} \in \operatorname{Euler}(p), r \in \operatorname{Euler}(q)$ as follows:

(1) $C_{p, r} \otimes C_{q, r}:=C_{p, r^{\prime}} \otimes\left(b_{i, j}\right)_{q \times q}=\left(B_{i, j}\right)_{q \times q} \quad$ a matrix of matrixes, which is a square matrix of $p q$-order, where $C_{q, r}=\left(b_{i, j}\right)_{q \times q},\left(B_{i, j}\right)_{q \times q}=C_{p, r^{\prime}}\left((p)_{b_{i, j}}\right)$.

(2) $C_{p, 1} \otimes_{R} C_{q, r}:=C_{p, 1} \otimes_{\left(r_{i, j}\right)_{q \times q}}\left(b_{i, j}\right)_{q \times q}=\left(D_{i, j}\right)_{q \times q}$ a matrix of matrixes, which is a square matrix of $p q$-order, where $C_{q, r}=\left(b_{i, j}\right)_{q \times q},\left(D_{i, j}\right)_{q \times q}=C_{p, r_{i, j}}\left((p)_{b_{i, j}}\right)$.

We can write the combination of $R=\left(r_{i, j}\right)_{q \times q}$ and $C_{q, r}=$ $\left(b_{i, j}\right)_{q \times q}$ as a new matrix $C_{q, r}^{R}=\left(b_{i, j} \mid r_{i, j}\right)_{q \times q}$.It is easy to prove that

(1) $C_{p, 1} \cong C_{p, r \prime}$, for any ' $\in \operatorname{Euler}(p)$.

(2) $C_{p, r} \otimes C_{q, r} \cong C_{q, r}, \otimes C_{p, r}, \quad$ for any $\quad r^{\prime} \in$ $\operatorname{Euler}(p), r \in \operatorname{Euler}(q)$.

(3) $C_{p, 1} \otimes_{R} C_{q, r} \cong C_{p, 1} \bigotimes_{R} C_{q, 1}$ for any fixed $R$.

Thus, we conclude that for $p, q \geq 2$, classifying $C_{p, 1} \otimes_{R} C_{q, r}$ depends only on the classification of $R$.

Definition 2 For $p, q \geq 2$, the matrixes $R$ and $R^{\prime}$ are square matrixes of q-order on Euler $(p)$, satisfying the diagonal congruence w.r.t. $p$., we call that $R, R^{\prime}$ have the same type, denote as $R \bowtie R^{\prime}$, if $C_{p, 1} \otimes_{R} C_{q, 1} \cong C_{p, 1} \otimes_{R^{\prime}} C_{q, 1}$.

\section{BASIC STRUCTURES OF FINITE GROUPS}

It is known that between complete Y-matrixes and finite groups are one-to-one. We can investigate structures of finite groups by complete Y-matrixes.

Lemma 4 (1) For any natural number $p \geq 2$, a cyclic group of $\mathrm{p}$-order is isomorphic to a group defined by the complete $\mathrm{Y}$ matrix $C_{p, 1}$.

(2) Let $G$ be a group of n-order $(n \geq 2)$, where $n=$ $p q(p, q \geq 2)$, and both $p$ and $q$ are prime numbers. Then there is a matrix $R=\left(r_{i, j}\right)_{q \times q}$ of row-rotations parameters, where $r_{i, j} \in \operatorname{Euler}(p)$, such that $T_{G} \cong C_{p, 1} \bigotimes_{R} C_{q, 1}$, where $T_{G}$ is the computation table of group $G$, and the unit element is located at the main diagonal of $T_{G}$.

Proof: (1) Let $G$ be a cyclic group of $p$-order $(p \geq 2)$, and let a generator of $G_{i}$ Then, $\left(e, a, a^{2}, \cdots, a^{p-1}\right)\left(e=a^{p}\right)$ is a cyclic sequence of symbols, where $e$ is the unit element of $G$. Clearly, the computation table of group $G$ is isomorphic to $C_{p, 1}$, and then $G$ is isomorphic to a group defined by the complete $\mathrm{Y}$ matrix $\mathrm{C}_{\mathrm{p}, 1}$.

(2) Let $G$ be a group of $n$-order $(n \geq 2)$, and let $p$ be a prime factor of $n, n=p q(p, q \geq 2)$. 
Then, there is an element $a$ of $p$-order. We take a as a generator, and construct a cyclic group $H_{1}$ of $p$-order. Without loss of generality (w.l.o.g.), we write $H_{1}=\left\{e, a_{1}, \cdots, a_{p-1}\right\}$, where $e$ is the unit element of $G$, and $a_{1}$ is the generator of $H$, $a_{i}=a_{1}^{i}(i=1,2, \cdots, p-1)$ and $a_{1}^{p}=e$.

Further, there are $(q-1)$ elements, $b_{2}, b_{3}, \cdots, b_{q}$ in $G$, such that

(1) $H=\left\{b_{1}=e, b_{2}, \cdots, b_{q}\right\}$ is a subgroup of $G$.

(2) $\left\{H_{1}, \cdots, H_{q}\right\}$ is a partition of $G$, where $H_{k}=b_{k} H_{1}=$ $\left\{b_{k} a: a \epsilon H_{1}\right\}(k=2, \cdots, q), G=\bigcup_{k=1}^{q} H_{k}$ and $H_{i} \cap H_{j}=\emptyset$ for any $1 \leq i \neq j \leq q$. Based on $H_{1}, \cdots, H_{q}$, we get $q$ cyclic sequence of symbols: $\left(A_{1}\right)=\left(e, a_{1}, a_{1}^{2}, \cdots, a_{1}^{p-1}\right)=$ $\left(a_{1,1}, a_{1,2}, \cdots, a_{1, p}\right) \quad$ and $\quad\left(A_{k}\right)=\left(b_{k}, b_{k} a_{1}, \cdots, b_{k} a_{1}^{p-1}\right)=$ $\left(a_{1,(k-1) p+1}, a_{1,(k-1) p+2}, \cdots, a_{1, k p}\right) k=2,3, \cdots, q$.

We can define a complete Y-matrix $C_{p, 1}$, and normalize $C_{p, 1}$ into $C_{p, p-1}$, where the unit $e$ is located at main diagonal of $C_{p, p-1}$. Please note for each $2 \leq k \leq q$ that $H_{k}$ has such closure property: $b_{k} a, b_{k} b \in H_{k}$ for any $a, b \in H_{1}$. Thus, we can get a complete Y-matrix $B_{k}$ and $B_{k} \cong C_{p, 1}$.

On the other hand, the subgroup $H=\left\{e, b_{2}, \cdots, b_{q}\right\}$ decides a CY-matrix. Since $q$ is a prime number, the group $H$ is a cyclic group. So, it is isomorphic to $C_{q, 1}$. Take $B_{1}, B_{2}, \cdots, B_{q}$ as elements, one can construct a group $C_{q}^{*}$ to be isomorphic to $C_{q, 1}$, and the expansion of $C_{q}^{*}$ is isomorphic to $G$. Therefore, there is a matrix $R=\left(r_{i, j}\right)_{q \times q}$ of row-rotations parameters, where $r_{i, j} \in \operatorname{Euler}(p)$, such that $T_{G} \cong C_{p, 1} \bigotimes_{R} C_{q, 1}$, where $T_{G}$ is the computation table of group $G$, and the unit element is located at the main diagonal of $T_{G}$.

\section{CONCLUSIONS AND FUTURE WORKS}

The matrix representation of a finite group is a complete $\mathrm{Y}$ matrix, and a complete Y-matrix decides a finite group. The "Four Endpoints Rule (FER)" is main geometric characterization of complete Y-matrixes. In this paper, we have discussed relations between the three (ordinary finite group, permutation group, and matrix group), and presented some basic structures and construction methods of finite groups. It is helpful for classifying and decomposing finite groups. The methods deal only with rotation of rows in matrixes. Further, we can consider the following method to construct finite groups.

(1) Combining rotations of rows and columns in matrixes.

(2) Combining cross rotations of rows and columns in matrixes.

Based on combination of factorization factors of natural number $\mathrm{n}$ and rotations, ones can construct finite groups with different structures.

\section{ACKNOWLEDGEMENTS}

The research work was supported by National Natural Science Foundation of China unNBder Grant No. 61262006, Major Applied Basic Research Program of Guizhou Province under Grant No.JZ20142001 and Science and Technology Foundation of Guizhou Province under Grant No.20122125.

\section{REFERENCES}

[1] Y. Cao, Introduction to Y Group. Science Press, Beijing (2012).

[2] A. Baker, Representations of Finite Groups, http: //www.maths.gla.ac.uk/ajb

[3] J. L. Alperin \& R. B. Bell, Groups and Representations, Springer-Verlag (1995).

[4] J. B. Fraleigh, A First Course in Abstract Algebra, 5th Edition, AddisonWesley (1994).

[5] G. James \& M. Liebeck, Representations and Characters of Groups, Cambridge University Press (1993).

[6] J.P. Serre, Linear Representations of Finite Groups, Springer-Verlag (1977).

[7] S. Sternberg, Group theory and physics, Cambridge University Press (1994).

[8] R. Solomon, On Finite Simple Groups and Their Classification, Notices American Mathematical Society. 42, 231-239, 1995.

[9] Batten, Lynn Margaret, Combinatorics of Finite Geometries, Cambridge University Press (1997). 